OPEN

SUBJECT AREAS:

STATISTICAL PHYSICS

NONLINEAR PHENOMENA

Received

1 August 2014

Accepted

3 October 2014

Published

27 October 2014

Correspondence and requests for materials should be addressed to

Z.-G.H. (huangzg@ Izu.edu.cn)

\title{
Universal flux-fluctuation law in small
} systems

\author{
Zi-Gang Huang ${ }^{1,2}$, Jia-Qi Dong ${ }^{1}$, Liang Huang ${ }^{1,2} \&$ Ying-Cheng Lai ${ }^{2,3}$
}

'Institute of Computational Physics and Complex Systems and Key Laboratory for Magnetism and Magnetic Materials of MOE, Lanzhou University, Lanzhou 730000, China, ${ }^{2}$ School of Electrical, Computer and Energy Engineering, Arizona State University, Tempe, AZ 85287, USA, ${ }^{3}$ Department of Physics, Arizona State University, Tempe, Arizona 85287, USA.

The relation between flux and fluctuation is fundamental to complex physical systems that support and transport flows. A recently obtained law predicts monotonous enhancement of fluctuation as the average flux is increased, which in principle is valid but only for large systems. For realistic complex systems of small sizes, this law breaks down when both the average flux and fluctuation become large. Here we demonstrate the failure of this law in small systems using real data and model complex networked systems, derive analytically a modified flux-fluctuation law, and validate it through computations of a large number of complex networked systems. Our law is more general in that its predictions agree with numerics and it reduces naturally to the previous law in the limit of large system size, leading to new insights into the flow dynamics in small-size complex systems with significant implications for the statistical and scaling behaviors of small systems, a topic of great recent interest.

A basic and important quantity characterizing a large variety of complex dynamical systems in the real world is flow or flux. For example, in a transportation network traffic flux is the main quantity of interest; in the Internet the flux of packets is key to information propagation and spreading; in an electrical power grid the normal flow of electricity is indication of system's designed operation; and in a neuronal network the flux of electrical pulses is responsible for information processing and ultimately for all kinds of biological functions. Due to the complex nature of the systems and inevitable presence of noise, in general the flux tends to fluctuate about its average in time intervals in which the system may be regarded as stationary.

In the study of complex dynamical systems, a fundamental question of continuous interest is whether there exists a universal law between the average flux and the fluctuation ${ }^{1-7}$. In this regard, an early work revealed a power-law (algebraic) relation between the two with the exponent taking on a finite set of discrete values, such as $1 / 2$ or $1^{1}$. Subsequently it was suggested ${ }^{3}$ that the power-law exponent can assume continuous values in the range $[1 / 2,1]$. Quite recently, the notion of power-law scaling between the fluctuation and the average flux was refuted and a non-algebraic type of relation between the two quantities was obtained ${ }^{6}$, with support from both model simulations and data from a realistic communication network system. In particular, let $\left\langle f_{i}\right\rangle$ be the average flux and $\sigma_{i}$ be the amount of fluctuation, the non-algebraic type of flux-fluctuation law is given by ${ }^{6}$

$$
\sigma_{i}=\sqrt{\left\langle f_{i}\right\rangle+\alpha^{2}\left\langle f_{i}\right\rangle^{2}},
$$

where $\alpha \equiv \sigma_{\text {ext }} /\langle M\rangle$ is a single parameter determined by the property of the external driving $M(t)$ only, $\sigma_{\text {ext }}$ and $\langle M\rangle$ are the standard deviation and the expectation value of $M(t)$, respectively. The discovery of Eq. (1) is important as it reveals a simple but definite relation between the flux and fluctuations, in spite of the complex nature of the underlying physical system.

In spite of the success of Eq. (1) in explaining the flux-fluctuation relation observed from certain real systems, there exists a paradox. In particular, Eq. (1) implies that, as the flux increases continuously, so would the fluctuation. Consider, for example, a complex network of small size, where the total amount of traffic, or flux, is finite. In such a system, the traffic flow through various nodes will exhibit different amount of fluctuations, depending on the corresponding flux. In the special but not unlikely case where most of the traffic flow passes through a dominant node in the network, the flux is large but the fluctuation observed from it must be small, since the total amount of traffic is fixed. For other nodes in the network the opposite would occur. Thus, in a strict sense, Eq. (1) is applicable only to physical systems of infinite size with less flux heterogeneity. An example of the failure of Eq. (1) for a small system is demonstrated in Fig. 1 panels (a) and (b), where the flux-fluctuation relation obtained from a real Microchip system ${ }^{1}$ is shown. We see that, on a logarithmic scale [see panel (a)], the flux- 

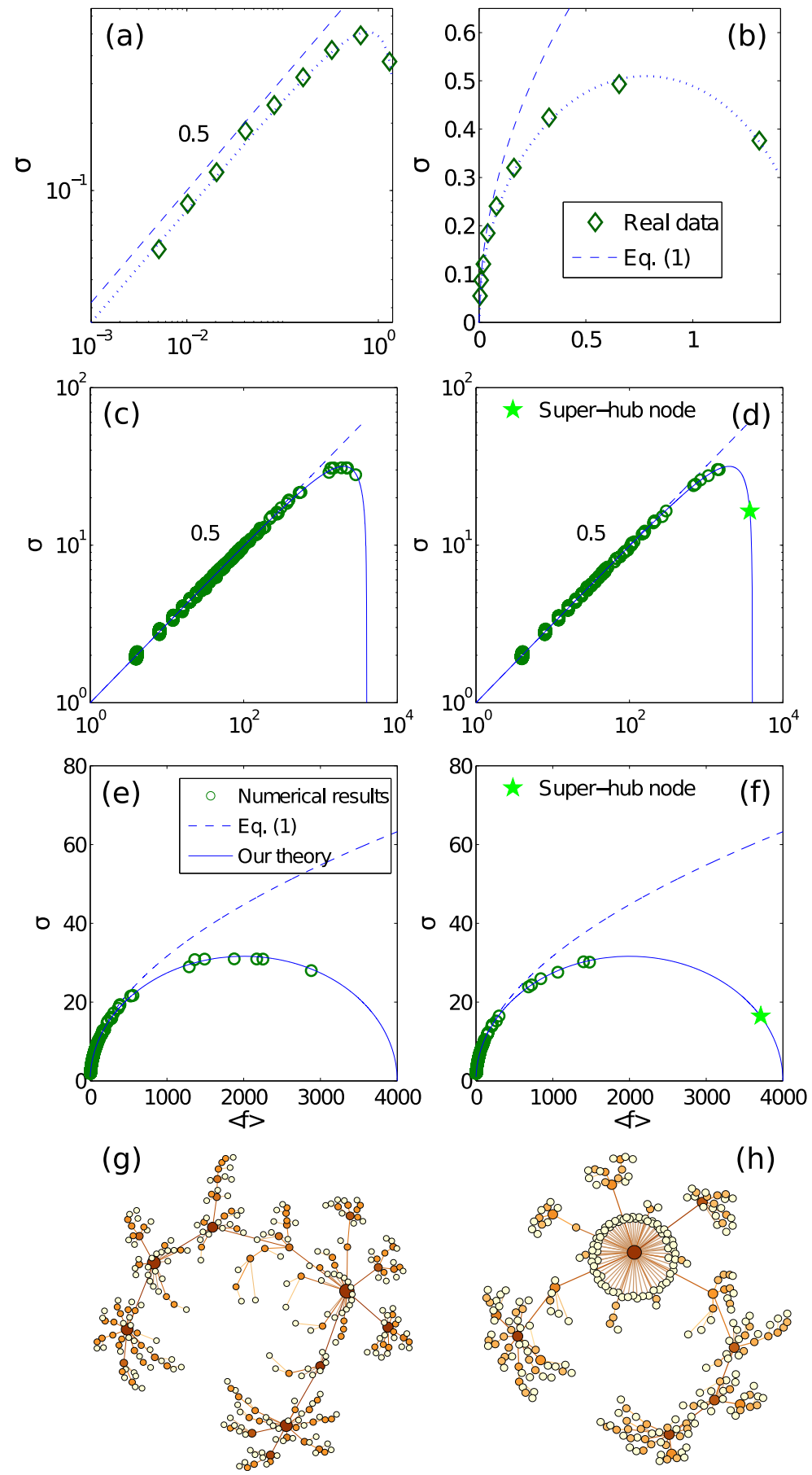

Figure 1 Empirical and numerical evidences for the breakdown of the previous flux-fluctuation law Eq. (1). Nodal flux fluctuation $\sigma_{i}$ versus the average flux $\left\langle f_{i}\right\rangle$, (a) and (b) for real data from microchips ${ }^{1}$, and $(c, d)$ and $(e, f)$ for the numerical data from heterogeneous networks on logarithmic and linear scales, respectively. The dashed and solid curves are the predictions of the previous flux-fluctuation law Eq. (1) and our new theory Eq. (2), respectively. And the dotted curves in $(\mathrm{a}, \mathrm{b})$ are fitting of real data which can be explained by our theory. Numerical simulations are realized on scale-free networks of size $N=2000$ and mean degree $\langle k\rangle=2$, under constant external driving $M=4000$. The networks of (c,e) and (d,f) are schematically illustrated in $(\mathrm{g})$ and $(\mathrm{h})$, respectively.

fluctuation behavior from the real data (open diamonds) appears to mostly agree with the prediction of Eq. (1) (dashed curve). However, as indicated in panel (b), on a linear scale there is substantial deviation of the real behavior from the prediction of Eq. (1). A careful examination of the flux-fluctuation relation for model network systems, especially for small systems with weak external driving or those with a high nodal flux heterogeneity, reveals that the deviation from the theoretical prediction is systematic. Curiosity demands that we ask the following question: in small complex physical system, is there a general flux-fluctuation law?

In this paper, we uncover a universal flux-fluctuation law in small systems. Taking into account realistic physical effects such as the contribution of internal randomness and combined effects of multiple external driving, we may obtain more accurate flux-fluctuation relation agreeing well to real data and numerical results (dotted and solid curves in Fig. 1). A key to our success lies in distinguishing the 
external driving from the intrinsic fluctuations in terms of their respective contributions to the interplay between flux and fluctuation, where the former represents the systematic or random perturbation upon the system from the outside world and determines the total flux of the system in any given time period. For example, in a river network, the external drive can be the precipitation in the basin region; for Internet or the urban traffic systems, external drive can be the daily rhythmic behavior of human activities ${ }^{8}$, such as the traffic in a city introduced by human's commuting between place of residence and place of work. Intrinsic fluctuations of the system, however, are generated by randomness in processes such as package generating, source-destination selection, path selection, and asymmetry of the underlying network topology, etc. We show that, for a small system, the effects of internal randomness on the flux-fluctuation interplay must be included and treated properly, and this leads to a nontrivial correction to Eq. (1) and results in a new law that is generally applicable to complex systems of small size and reduces to Eq. (1) in the limit of large system size. Since realistic physical systems are finite, especially considering that the statistical physics of small systems are of great recent interest ${ }^{9-11}$, we expect our result to be appealing to the broader scientific community.

\section{Results}

Here we give the numerical evidence of systematic breakdown of Eq. (1) and validity of new flux-fluctuation law,

$$
\sigma_{i}^{2}=\left\langle f_{i}\right\rangle+\left[\alpha^{2}-\frac{1}{\langle M\rangle}\right]\left\langle f_{i}\right\rangle^{2}
$$

with the theoretical analysis of which given in Sec. Methods. Compared with the previously obtained ${ }^{1-7}$ flux-fluctuation law [Eq. (1)], Eq. (2) has the additional term $-\left\langle f_{i}\right\rangle^{2} /\langle M\rangle$, which accounts for the contribution to the fluctuations by the internal randomness.

Flux-fluctuation law in scale-free networks. To provide more evidence for the breakdown of Eq. (1) in physical systems, we consider scale-free networks ${ }^{12}$ and implement packet-flow dynamics ${ }^{13,14}$. At time step $t$, the system generates $M(t)$ packets. Each packet is assigned a pair of randomly selected source and destination. Packets are delivered step-by-step through the respective shortest paths from their sources to destinations. Here, the external drive can be characterized by $M(t)$. We firstly consider the simple case of constant external drive $M$ (i.e., $\sigma_{\text {ext }}=0$ ), under which Eqs. (1) and (2) correspond to $\sigma_{i}^{2}=\left\langle f_{i}\right\rangle$ and $\sigma_{i}^{2}=\left\langle f_{i}\right\rangle-\left\langle f_{i}\right\rangle^{2} /\langle M\rangle$, respectively. The constant external drive is ubiquitous in real systems, e.g., the river flow replenished by the confined groundwater, and stable flow on Internet introduced by the pre-installed task processing programs (which are not restricted to the daily surfing rhythm of users). Effective constant drive also takes place in the networked system composed of nodes with limited delivery capacity. For example, a node with saturated flow will deliver a constant number of packets at each time step, which thus acts as a constant external drive to the downstream subsystem. Constant external drive also exists in closed traffic systems, such as the blood circulation systems of animals and water heating system for buildings. Furthermore, the large observation time scale to traffic systems ${ }^{3-7}$ also give rise to the situation of a constant external drive, in case that the window size exceeds the characteristic period and thus the detailed temporal information of the traffic flow is bypassed effectively.

Figure 1(c) shows, on a logarithmic scale, the fluctuation $\sigma_{i}$ versus the average flux $\left\langle f_{i}\right\rangle$ for various nodes, where $M=4000$, the network size is $N=2000$, the dashed line is from Eq. (1), the solid curve is from our theory, and the open circles are from simulation. Figure 1(e) shows the same data but on a linear scale. For the simulation results, the error bars associated with the points obtained from different realizations of the dynamics are smaller than the symbol size. One typical realization of the network structure is shown in Fig. 1(g). We see that the failure of Eq. (1) mainly occurs in the large $\left\langle f_{i}\right\rangle$ regime. To further illustrate the deviation, we consider the extreme case where most of the network traffic is through a superhub node so that $\left\langle f_{i}\right\rangle \approx M$. The resulting flux-fluctuation relation is shown in Figs. 1(d) and (f), and the corresponding network structure is shown in Fig. 1(h). We see that the data point specifying the fluxfluctuation of the super-hub node, marked by a star, deviates significantly from Eq. (1) in that the fluctuation amount is small, but it still falls on our theoretical curve. Intuitively, this can be understood by noting that, because the external drive is constant, flux fluctuations are caused entirely by the intrinsic randomness that is minimal for the super-hub node. In a realistic physical system, the components bearing relatively large amounts of flux tend to have small fluctuations due to the boundedness of the total amount of flux in the system, a feature that Eq. (1) fails to incorporate.

Flux-fluctuation law in small systems. We next test networked systems of much smaller size, which are ubiquitous in the physical world such as functional biological networks composed of a few proteins, quantum communication networks of a limited number of quantum repeaters, and local ad hoc computer/communication networks supporting a small group of users. For a variety of combinations of (small) network size and mean degree, we obtain essentially the same results as in Fig. 1: large deviations from the previous flux-fluctuation law [Eq. (1)] and excellent agreement with our theory. Representative examples are shown in Fig. 2 for a number of networked systems, all of $N=50$ nodes but with different structural parameters. A constant external drive with parameter $M=$ 4000 and the shortest path-length routing protocol are used. Plotted in each panel in Fig. 2 are predictions from Eq. (1) (dashed curves) and from our theory Eq. (2) (solid curves), as well as simulation results (open circles). We see that, for small systems, the disagreement between numerics and Eq. (1) becomes more drastic, especially for smaller mean-degree value. However, in all cases, our new law [Eq. (2)] agrees excellently with the corresponding numerical results, indicating the importance and necessity of including our correctional term $\left[-\left\langle f_{i}\right\rangle^{2} /\langle M\rangle\right]$ in the flux-fluctuation law for small sparse networked systems.

Validity of Eq. (2) with respect to network topologies and routing schemes. To demonstrate the general applicability Eq. (2), we carry out numerical simulations of packet-flow dynamics ${ }^{13,14}$ under a large number of combinations of complex-network topologies and routing schemes. Specifically, we use scale-free ${ }^{12}$, random ${ }^{15}$, and smallworld $^{16}$ networks, and routing protocols such as those based on shortest path-length, random-walk, and efficient-path scheme $(\mathrm{EPS})^{17}$. In all cases considered, there is excellent agreement between the numerics and Eq. (2). For example, Fig. 3 shows the results from random and small-world networks under the shortest path-length routing protocol. The small-world networks are generated by adding 5 links randomly on a regular onedimensional ring lattice of size $N=100$ and mean degree $c=4$. Interestingly, notwithstanding the intrinsic homogeneity underlying this standard class of small-world network model ${ }^{16}$, there is still wide spread of flux due to the inhomogeneous distribution of traffic flow caused by shortest path-length routing. Figure 4 shows the results of efficient-path routing scheme on scale-free and random networks. In this scheme, node $i$ in the network is weighted by $w_{i}=k_{i}^{\gamma}$, where $k_{i}$ is $i$ 's degree and $\gamma$ is a turnable parameter characterizing the routing strategy. A packet from source $j_{1}$ to destination $j_{2}$ will choose a route with the minimum sum of weights: $\sum_{i \in \sigma_{j_{1} j_{2}}} k_{i}^{\gamma}$, where $\sigma_{j_{1} j_{2}}$ denotes the path from $j_{1}$ to $j_{2}$. By varying the parameter $\gamma$, we can select certain subsets of nodes on the packet-transport path, such as hubs or small-degree nodes. From Fig. 4, we see that, while different routing schemes lead to large spread in the range of the flux 

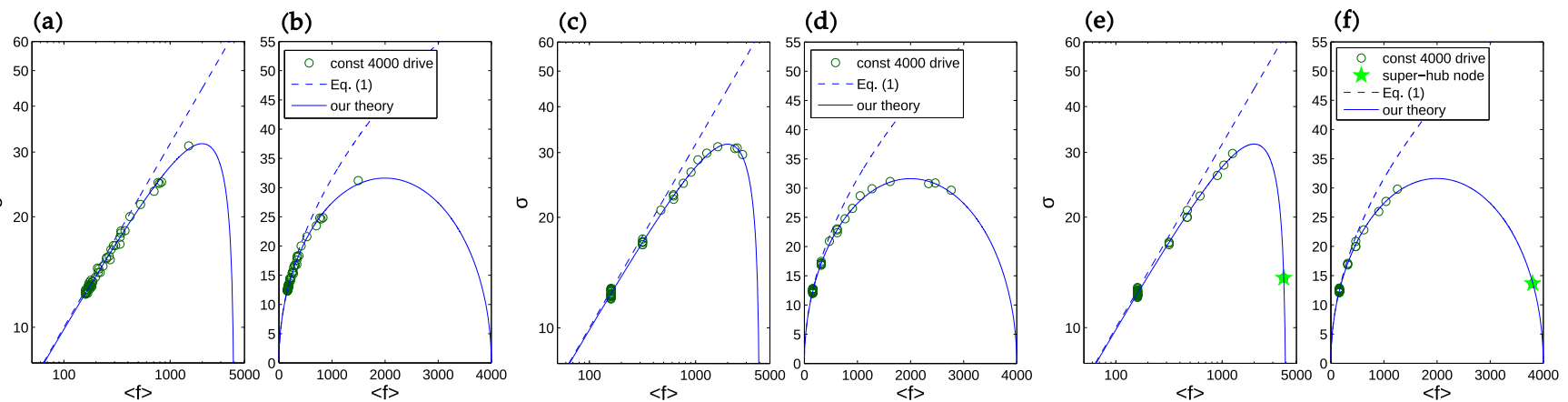

Figure $2 \mid$ Flux-fluctuation relationship in small systems. Nodal flux fluctuation $\sigma_{i}$ versus the average flux $\left\langle f_{i}\right\rangle$ for small networks of size $N=50$. The mean degree is $\langle k\rangle=4$ for $(\mathrm{a}, \mathrm{b})$ and $\langle k\rangle=2$ for $(\mathrm{c}, \mathrm{d})$. Panels $(\mathrm{e}, \mathrm{f})$ are results from a modified network of mean degree $\langle k\rangle=2$ but with a super-hub node. In panels $(\mathrm{a}, \mathrm{c}, \mathrm{e})$ the flux-fluctuation relations are plotted on a logarithmic scale while in panels $(\mathrm{b}, \mathrm{d}, \mathrm{f})$, a linear scale is used. The dashed curves are obtained from Eq. (1) and the solid curves are predictions from our modified flux-fluctuation law Eq. (2). All systems are under a constant external drive defined by $M=4000$.

values, the flux-fluctuation relations all collapse into the single curve predicted by our theory.

In all cases examined, we find that, insofar as the flux in the system is heterogeneous, regardless of the source of heterogeneity (e.g., network topology or routing scheme), our correctional term in Eq. (2) is absolutely necessary to account for the numerically calculated fluxfluctuation relations.

Time-dependent external driving. To further demonstrate the general validity of Eq. (2), we present examples of time-dependent random external driving $M(t)$, i.e., with nonzero $\sigma_{\text {ext }}^{2}$. Figure 5 shows, for three cases where $\langle M\rangle=4000$ but $\sigma_{\text {ext }}^{2}=0,10034$, and 49923, numerically obtained flux-fluctuation relations and the corresponding theoretical predictions from both Eqs. (1) and (2). Again, there is excellent agreement between our theory and the numerics. For the case where the external driving is more random, the contribution of the internal randomness [the first and third terms in Eq. (2)] is relatively small. In this case, the previous theory [Eq. (1)] agrees reasonably well with the numerics, as it is particularly suitable for situations where the external driving is highly fluctuating. Our theory is more general as it accurately includes the effects of both intrinsic and external randomness and is thus more broadly applicable. We have also considered flux through links and found that the link flux $f_{i j}$ also renders applicable Eq. (2).

Our formula, Eq. (2), also makes possible a detailed understanding of the effect of random external drive. Consider, for example, the special case where the sequence of the external driving $M(t)$ follows a Poisson distribution so that $\sigma_{\text {ext }}^{2}=\langle M\rangle$. In this case, Eq. (2) gives $\sigma_{i}^{2}=\left\langle f_{i}\right\rangle$. As the external driving has $\sigma_{\text {ext }}^{2}$ shifts away from $\langle M\rangle$, no matter how small, the nodal fluctuation immediately becomes a nonlinear function of the average flux. For $\left.\sigma_{\text {ext }}^{2}\right\rangle\langle M\rangle\left(\sigma_{\text {ext }}^{2}<\langle M\rangle\right)$, fluctuations at the large-flux nodes are relatively larger (smaller), with the quadratic coefficient in Eq. (2) being positive (negative). We note that, in the previous formula Eq. (1), the coefficient in the quadratic

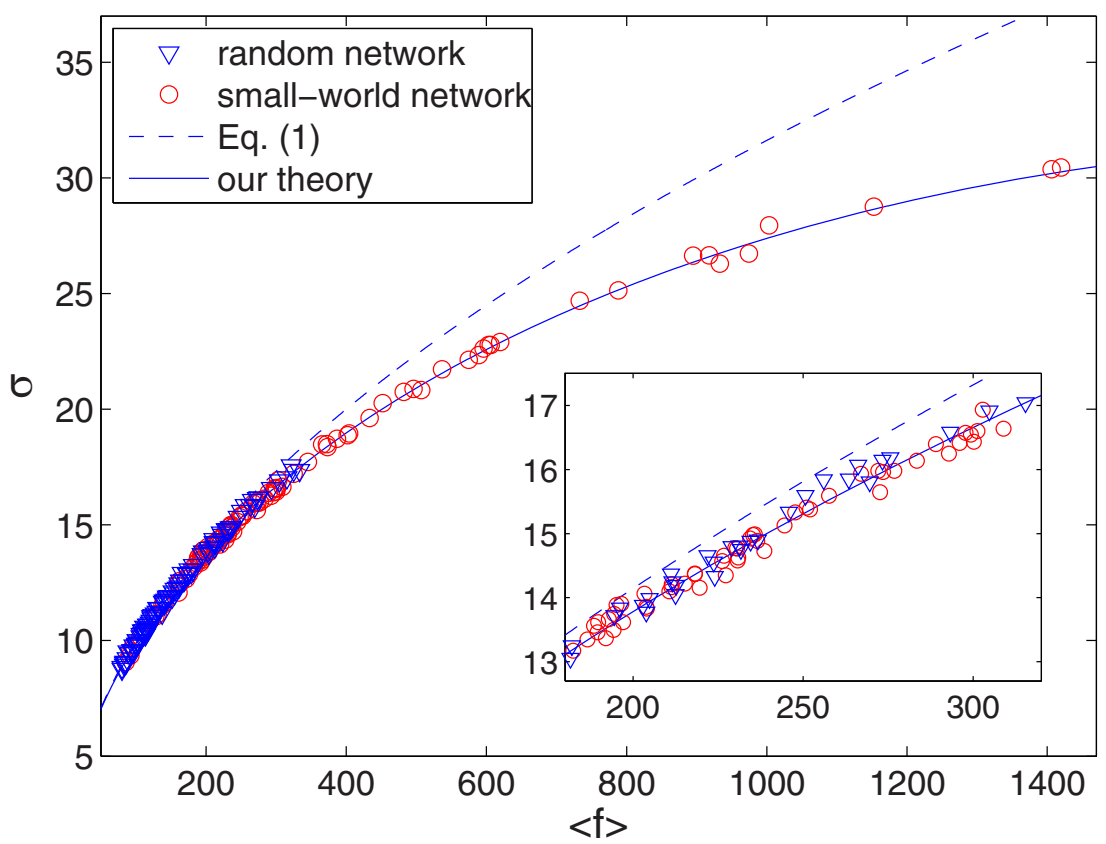

Figure $3 \mid$ Flux-fluctuation relationship on different network topologies. Nodal flux fluctuation $\sigma_{i}$ versus average flux $\left\langle f_{i}\right\rangle$ for networks of different topologies but of the same size $N=100$. The mean degrees $\langle k\rangle$ of random and small-world networks are 5 and 4 , respectively. The dashed and solid curves are predictions of Eq. (1) and Eq. (2), respectively. The constant external drive has strength $M=4000$. The subgraph is the magnification of the small-flux regions. 

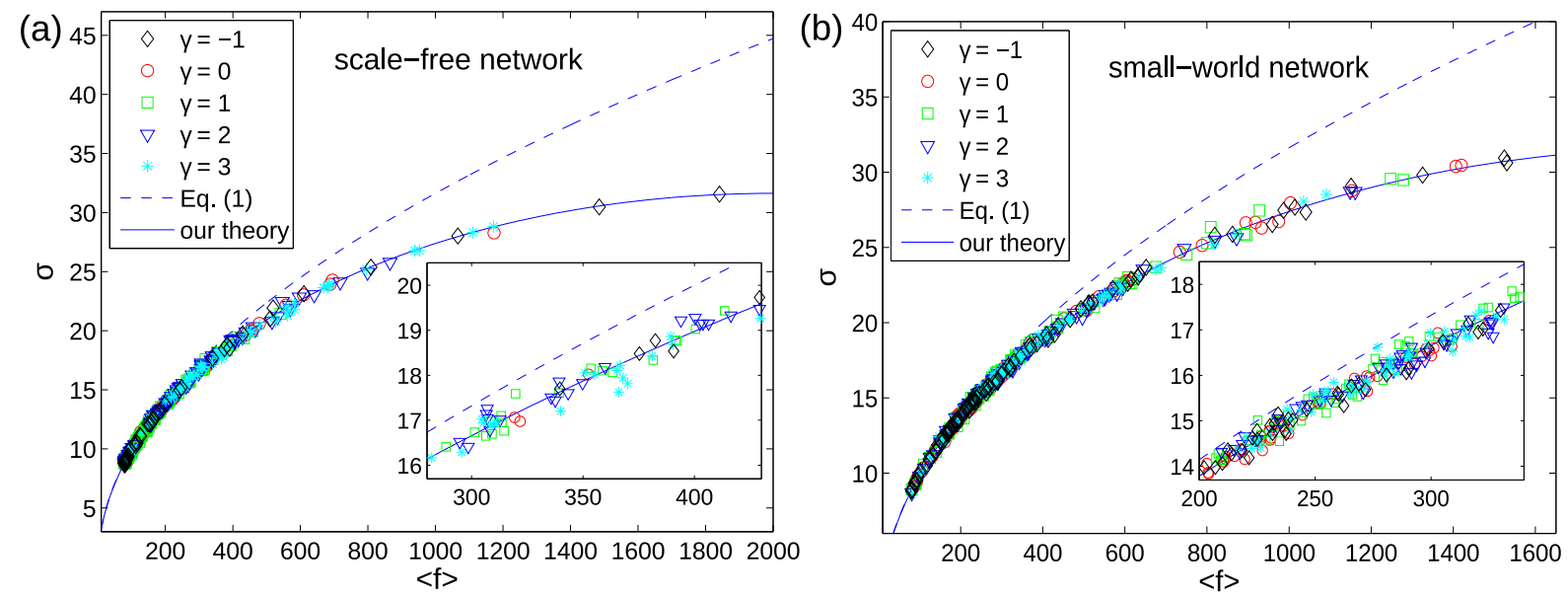

Figure $4 \mid$ Flux-fluctuation relationship under different routing schemes. Nodal flux fluctuation $\sigma_{i}$ versus the average flux $\left\langle f_{i}\right\rangle$ for $(\mathrm{a})$ scale-free networks and (b) small-world networks of size $N=100$ and mean degree $\langle k\rangle=4$ under efficient-path routing schemes. The dashed and solid curves are predictions of Eq. (1) and Eq. (2), respectively. The constant external drive has strength $M=4000$. The subgraphs are the respective magnifications of the small-flux regions.

term is always positive, whereas in our formula [Eq. (2)], this coefficient can take on either positive or negative values, depending on the degree of randomness of the external driving. An example of negative coefficient from real-world systems is shown in Figs. 1(a)(b).

\section{Discussion}

To summarize, we argue and demonstrate that the previously obtained flux-fluctuation law is valid but in principle only for large complex physical systems. Especially, among others it predicts continuously enhanced fluctuations as the average flux is increased, a situation that cannot occur in small physical systems under finite external drive. The failure of this law is demonstrated using both real and model systems. By analyzing the validity of the mathematical assumption for the probability distribution of the flow dynamics, we derive a general flux-fluctuation law, which correctly predicts the possible maximized fluctuation for intermediate flux. Our law includes the previously obtained flux-fluctuation law as a special case, and it is universally applicable to all kinds of complex dynamical systems, large or small, homogeneous or heterogeneous. One immediate application of our flux-fluctuation law is to understand the dynamics of extreme events on complex systems ${ }^{18,19}$, where simultaneous occurrence of many extreme events can have devastating consequences on the functions of the corresponding networks. The flux-fluctuation law can be used to forecast the number of extreme events on the network, providing guidance to articulating control strategies to suppress extreme events ${ }^{19}$. The flux-fluctuation relation is a fundamental characteristic of any complex system. The new law presented in this paper can provide insights into the various dynamical processes on small complex systems, and this can be important in view of the growing recent interest in the statistical mechanics of small systems in physics, chemistry, and biology ${ }^{9-11}$.

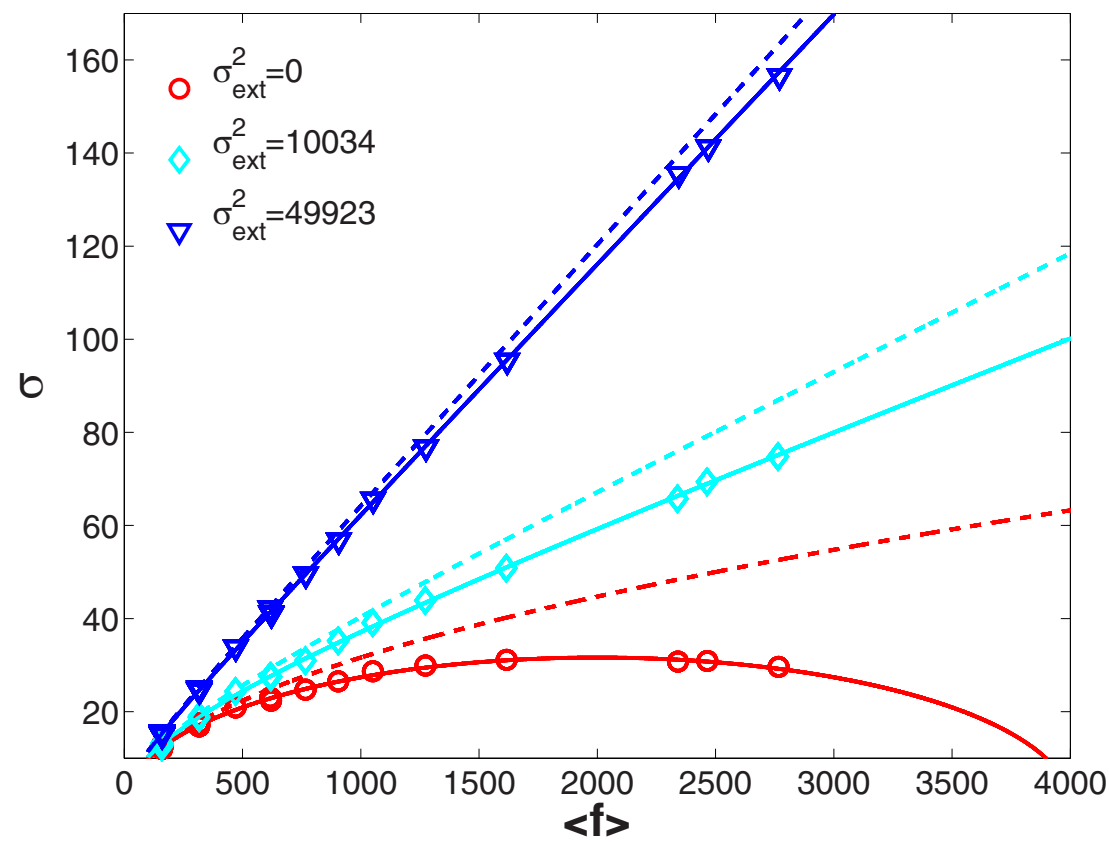

Figure 5 Flux-fluctuation relationship under time-dependent external drive. Nodal flux fluctuation $\sigma_{i}$ versus the average flux $\left\langle f_{i}\right\rangle$ for external drive with three values of $\sigma_{\text {ext }}$ (from bottom: 0, 10034, and 49923) but identical mean driving strength $\langle M\rangle=4000$. The dashed and solid curves respectively are the predictions of Eq. (1) and Eq. (2). The scale-free network is of size $N=50$ and mean degree $\langle k\rangle=2$. 


\section{Methods}

Here, we derive a general flux-fluctuation law for small physical systems. The probability for one given packet to visit node $i$ with $k_{i}$ links is $p_{i}=k_{i} / 2 E$ in the stationary regime ${ }^{6}$, where $E$ is the total number of links and $\sum_{i} p_{i}=1$. If the system is under a constant external drive $M$, the expectation value of the flux at $i$ is $\left\langle f_{i}\right\rangle=M p_{i}$. In the previous derivation of Eq. (1), the probability of $f_{i}=n$ is assumed to follow the Poisson distribution $P_{\pi}\left(n, \lambda_{i}\right)=e^{-\lambda_{i}} \lambda_{i}^{n} / n$ ! with $\lambda_{i}=M p_{i}$. We note, however, that this assumption is not proper for heterogeneous, small size systems where the probability distribution is actually binomial. The Poisson distribution is an asymptotic form of the binomial distribution in the limit of $M \rightarrow \infty$ for fixed $M p_{i}$. Here, $p_{i}$ is related to the heterogeneity and the size $N$ of the system. For example, for a highly heterogeneous networked system, the value of $p_{i}$ associated with super-hubs can be large. For small networks, all nodes are with relatively large values of $p_{i}$. In both cases, the assumption of Poisson distribution cannot be properly justified. Our hypothesis is then that, the probability that node $i$ gets $n$ packets obeys a binomial distribution: $P_{\pi}(n, M)=C_{M}^{n} p_{i}^{n}\left(1-p_{i}\right)^{M-n}$, where $M$ is the total number of packets in the system. For the case where the external driving is time-dependent, the number $M$ itself follows some probability distribution, denoted by $P_{\text {ext }}(M)$. We thus have

$$
\begin{aligned}
P_{f_{i}}(n) & =\sum_{M=0}^{\infty} P_{\pi}(n, M) P_{\mathrm{ext}}(M) \\
& =\sum_{M=0}^{\infty} C_{M}^{n} p_{i}^{n}\left(1-p_{i}\right)^{M-n} P_{\mathrm{ext}}(M) .
\end{aligned}
$$

The average flux is given by

$$
\begin{aligned}
\left\langle f_{i}\right\rangle & =\sum_{n=0}^{\infty} n P_{f_{i}}(n)=\sum_{n=0}^{\infty} n \sum_{M=0}^{\infty} P_{\pi}(n, M) P_{\text {ext }}(M) \\
& =\sum_{M=0}^{\infty}\left[\sum_{n=0}^{\infty} n P_{\pi}(n, M)\right] P_{\text {ext }}(M)=p_{i}(M),
\end{aligned}
$$

where $\langle M\rangle=\sum_{M=0}^{\infty} M P_{\text {ext }}(M)$ and $\left\langle M^{2}\right\rangle=\sum_{M=0}^{\infty} M^{2} P_{\text {ext }}(M)$ are respectively the first and second moments of the external drive. The second-order moment of the flux is

$$
\begin{aligned}
\left\langle f_{i}^{2}\right\rangle & =\sum_{n=0}^{\infty} n^{2} P_{f_{i}}(n)=\sum_{M=0}^{\infty}\left[\sum_{n=0}^{\infty} n^{2} P_{\pi}(n, M)\right] P_{\text {ext }}(M) \\
& =\left(p_{i}-p_{i}^{2}\right)\langle M\rangle+p_{i}^{2}\left\langle M^{2}\right\rangle .
\end{aligned}
$$

The flux fluctuation of node $i$ can then be calculated as

$$
\begin{aligned}
\sigma_{i}^{2} & \equiv\left\langle f_{i}^{2}\right\rangle-\left\langle f_{i}\right\rangle^{2} \\
& =\left[\left(p_{i}-p_{i}^{2}\right)\langle M\rangle+p_{i}^{2}\left\langle M^{2}\right\rangle\right]-\left(p_{i}\langle M\rangle\right)^{2} \\
& =\left\langle f_{i}\right\rangle+\left\langle f_{i}\right\rangle^{2} \sigma_{\text {ext }}^{2} /\langle M\rangle^{2}-\left\langle f_{i}\right\rangle^{2} /\langle M\rangle .
\end{aligned}
$$

where $\sigma_{\text {ext }}^{2} \equiv\left\langle M^{2}\right\rangle-\langle M\rangle^{2}$ is the variance of the external driving. With $\alpha \equiv \sigma_{\text {ext }} /\langle M\rangle$, we obtain Eq. (2).

Equation (2) can be generalized to the situations where complex system is driven by multiple external inputs $M_{J}(t)(J=1,2, \ldots)$. Take the system with two external drives $M_{1}(t)$ and $M_{2}(t)$ as an example, the flux on one given node $i$ is $n=$ $n_{1}+n_{2}$, i.e., the sum of the two fluxes $n_{1}$ and $n_{2}$ due to the two external drives. The temporal behaviors of $n_{1}$ and $n_{2}$ on node $i$ are determined by the sequences of $M_{1}(t)$ and $M_{2}(t)$, as well as the respective fractions of the fluxes distributed on node $i$, denoted by $\left\{p_{i 1}\right\}$ and $\left\{p_{i 2}\right\}$ with $i \in[1, N]$. The variance of the total flux can be written as

$$
\begin{aligned}
\sigma_{n}{ }^{2} & \equiv\left\langle n^{2}\right\rangle-\langle n\rangle^{2} \\
& ={\sigma_{n_{1}}}^{2}+{\sigma_{n_{2}}}^{2}+2 \sigma_{n_{1}} \sigma_{n_{2}} C_{12} .
\end{aligned}
$$

where the two variances ${\sigma_{n_{1}}}^{2}$ and $\sigma_{n_{2}}{ }^{2}$ are given by Eq. (2) with the respective parameters of external drives, $\alpha_{J}$ and $\left\langle M_{J}\right\rangle(J=1,2)$. In Eq. (3), $C_{12}$ is the Pearson correlation coefficient between $n_{1}$ and $n_{2}$ defined as

$$
C_{12} \equiv\left[\left\langle n_{1} n_{2}\right\rangle-\left\langle n_{1}\right\rangle\left\langle n_{2}\right\rangle\right] / \sigma_{n_{1}} \sigma_{n_{2}} .
$$

For the case of independent $n_{1}$ and $n_{2}$, we have $C_{12}=0$ and so $\sigma_{n}{ }^{2}=\sigma_{n_{1}}{ }^{2}+\sigma_{n_{2}}{ }^{2}$. For the extreme case of $C_{12}=1$, e.g., $n_{1}=n_{2}$, we have $\sigma_{n}{ }^{2}=4 \sigma_{n_{1}}{ }^{2}$. For the other case $C_{12}=-1$, e.g., $n_{1}+n_{2}=$ const., we get $\sigma_{n}{ }^{2}=0$.

The correlation between $n_{1}$ and $n_{2}$ are intimately related to the correlation between the external drives $M_{1}(t)$ and $M_{2}(t)$. Independent $M_{1}(t)$ and $M_{2}(t)$ lead to independent $n_{1}$ and $n_{2}$ (i.e., $C_{12}=0$ ). In this case, Eq. (3) can be written as

$$
\begin{aligned}
\sigma_{n}^{2} & =\sigma_{n_{1}}^{2}+\sigma_{n_{2}}^{2} \\
& =\left\langle n_{1}\right\rangle+\left[\alpha_{1}^{2}-\frac{1}{\left\langle M_{1}\right\rangle}\right]\left\langle n_{1}\right\rangle^{2}+\left\langle n_{2}\right\rangle+\left[\alpha_{2}^{2}-\frac{1}{\left\langle M_{2}\right\rangle}\right]\left\langle n_{2}\right\rangle^{2} \\
& =\left\langle M_{1}\right\rangle p_{i 1}+\left[\frac{\sigma_{\text {ext }_{1}}^{2}}{\left\langle M_{1}\right\rangle^{2}}-\frac{1}{\left\langle M_{1}\right\rangle}\right]\left(\left\langle M_{1}\right\rangle p_{i 1}\right)^{2}+\left\langle M_{2}\right\rangle p_{i 2}+\left[\frac{\sigma_{\text {ext }_{2}}^{2}}{\left\langle M_{2}\right\rangle^{2}}-\frac{1}{\left\langle M_{2}\right\rangle}\right]\left(\left\langle M_{2}\right\rangle p_{i 2}\right)^{2} .
\end{aligned}
$$

In the following, we present a number of examples with independent $M_{1}(t)$ and $M_{2}(t)$, for which analytical formulas relating $\sigma_{n}{ }^{2}$ and $\langle n\rangle$ can be derived.

Independently and identically distributed external drives with identical values of $\left\{p_{i}\right\}$. If $M_{1}(t)$ and $M_{2}(t)$ are independently and identically distributed (IID) and $p_{i 1}=$ $p_{i 2}$, we have $\sigma_{\text {ext }_{1}}^{2}=\sigma_{\text {ext }_{2}}^{2},\left\langle M_{1}\right\rangle=\left\langle M_{2}\right\rangle$, and $\langle n\rangle=2\left\langle n_{1}\right\rangle$, leading to

$$
\begin{aligned}
\sigma_{n}{ }^{2} & =\left\langle n_{1}\right\rangle+\left\langle n_{2}\right\rangle+\left[\frac{\sigma_{\text {ext }_{1}}^{2}}{\left\langle M_{1}\right\rangle^{2}} \frac{1}{\left\langle M_{1}\right\rangle}\right] \cdot\left[\left\langle n_{1}\right\rangle^{2}+\left\langle n_{2}\right\rangle^{2}\right] \\
& =\langle n\rangle+\frac{1}{m} \cdot\left[\frac{\sigma_{\text {ext }}^{2}}{\left\langle M_{1}\right\rangle^{2}}-\frac{1}{\left\langle M_{1}\right\rangle}\right]\langle n\rangle^{2},
\end{aligned}
$$

where $m=2$ is the number of external drives. Equation (6) in fact gives the general flux-fluctuation law for the system under $m \in \mathrm{N}$ IID external drives. However, the identical distribution of external drives is not a necessary condition to obtain Eq. (6). Insofar as the external drives have the identical values of $\sigma_{\text {ext }}^{2}$ and $\langle M\rangle$ (identical first and second moments), Eq. (6) can be obtained.

Multiple independent drives with one constant drive. Suppose that $M_{2}(t)=M_{2}$ is a constant drive but its value of $\left\{p_{i}\right\}$ is identical to that of the time-dependent drive $M_{1}(t)$. We can then write Eq. (5) as

$$
\begin{aligned}
\sigma_{n}^{2}= & \left\langle M_{1}\right\rangle p_{i 1}+\left[\frac{\sigma_{\text {ext }_{1}}^{2}}{\left\langle M_{1}\right\rangle^{2}}-\frac{1}{\left\langle M_{1}\right\rangle}\right]\left(\left\langle M_{1}\right\rangle p_{i 1}\right)^{2} \\
& +M_{2} p_{i 2}-\frac{1}{M_{2}}\left(M_{2} p_{i 2}\right)^{2} \\
= & \langle n\rangle+\left[\frac{\sigma_{\text {ext }_{1}}^{2}}{\left[\left\langle M_{1}\right\rangle+M_{2}\right]^{2}}-\frac{1}{\left[\left\langle M_{1}\right\rangle+M_{2}\right]}\right]\langle n\rangle^{2},
\end{aligned}
$$

which is similar to case of a single external drive as described by Eq. (2), with $\sigma_{\text {ext }}^{2}=\sigma_{\text {ext }}^{2}+0$, and $\langle M\rangle=\left\langle M_{1}\right\rangle+M_{2}$. From Eqs. (6) and (7), we see that the case with $\left\{p_{i 1}\right\}=\left\{p_{i 2}\right\}$ preserves the form of our flux-fluctuation law.

Independent drives with different $\left\{p_{i}\right\}$. The situation of external drives with nonidentical parameters, $\left\{p_{i 1}\right\} \neq\left\{p_{i 2}\right\}$, becomes slightly more complicated. For example, from Eq. (5) we see that, if $\left\langle M_{1}\right\rangle=\left\langle M_{2}\right\rangle$, all nodes receiving the same amount of driving $\left(p_{i 1}+p_{i 2}\right)$ will have identical average flux $\langle n\rangle$, but the contributions of $\sigma_{\mathrm{ext}_{1}}^{2}$ and $\sigma_{\text {ext } 2}^{2}$ to the nodal fluctuations of these nodes will depend on the relative values of $p_{i 1}$ and $p_{i 2}$. The nodes with identical value of $\langle n\rangle$ may have different values of $\sigma_{n}{ }^{2}$. From this simple example, we can see that, the single-valuedness of the relation between fluctuation and average flux cannot be guaranteed for external drives with $\left\{p_{i 1}\right\} \neq\left\{p_{i 2}\right\}$.

Here, we consider a special case for which a single-valued flux-fluctuation relation can be analytically obtained. Suppose $M_{2}(t)=M_{2}$ is a constant external drive providing a background flux to all the nodes, i.e., it distributes the flux homogeneously among nodes with probability $p_{i 2}=1 / N$. We can rewrite Eq. (5) as

$$
\begin{aligned}
\sigma_{n}{ }^{2} & =\left\langle n_{1}\right\rangle+\left[\frac{\sigma_{\mathrm{ext}_{1}}^{2}}{\left\langle M_{1}\right\rangle^{2}}-\frac{1}{\left\langle M_{1}\right\rangle}\right]\left\langle n_{1}\right\rangle^{2}+\frac{M_{2}}{N}-\frac{M_{2}}{N^{2}} \\
& =b\langle n\rangle+a\langle m\rangle^{2}+c,
\end{aligned}
$$

where

$$
\begin{aligned}
& \langle n\rangle=\left\langle n_{1}\right\rangle+\frac{M_{2}}{N}, \\
& a=\frac{\sigma_{\text {ext }_{1}}^{2}}{\left\langle M_{1}\right\rangle^{2}}-\frac{1}{\left\langle M_{1}\right\rangle}, \\
& b=1-2\left[\frac{\sigma_{\text {ext }_{1}}^{2}}{\left\langle M_{1}\right\rangle^{2}}-\frac{1}{\left\langle M_{1}\right\rangle}\right] \frac{M_{2}}{N}, \\
& c=\left[\frac{\sigma_{\text {ext }_{2}}^{2}}{\left\langle M_{1}\right\rangle^{2}}-\frac{1}{\left\langle M_{1}\right\rangle}\right]\left(\frac{M_{2}}{N}\right)^{2}-\frac{M_{2}}{N^{2}},
\end{aligned}
$$

and the constant external drive $M_{2}$ distributes flux to all the nodes with equal probability. It not only adds a constant term to the mean flux of the nodes, but also introduces fluctuation to each node.

For the deterministic case where the drive $M_{2}$ contributes $M_{2} / N$ amount of flux but without fluctuation to each node, we have 


$$
\begin{aligned}
\sigma_{n}{ }^{2} & =\left\langle n_{1}\right\rangle+\left[\frac{\sigma_{\text {ext }}^{2}}{\left\langle M_{1}\right\rangle^{2}}-\frac{1}{\left\langle M_{1}\right\rangle}\right]\left\langle n_{1}\right\rangle^{2} \\
& =b\langle n\rangle+a\langle n\rangle^{2}+c^{\prime},
\end{aligned}
$$

where

$$
c^{\prime}=\left[\frac{\sigma_{\mathrm{ext}_{1}}^{2}}{\left\langle M_{1}\right\rangle^{2}}-\frac{1}{\left\langle M_{1}\right\rangle}\right]\left(\frac{M_{2}}{N}\right)^{2}-\frac{M_{2}}{N} .
$$

We see that, the stable background flux introduces a nonzero constant term $c$ (or $c^{\prime}$ ) into the flux-fluctuation law. Also, the linear coefficient $b$ is no longer limited to unity. In this case, the functional form of $\sigma_{n}{ }^{2}$ versus $\langle n\rangle$ has three coefficients, where the number of system parameters, i.e., $\sigma_{\text {ext }}^{2},\left\langle M_{1}\right\rangle$, and $M_{2}$, are also three. In a real traffic system under identical background input to each node and a time-varying external drive, the parameters of the drives can be derived according to the values of $a, b$, and $c$ or $c^{\prime}$, which can be obtained from a parabolic fit to the actual flux-fluctuation data.

In addition, the behavior of flux fluctuation from real data, such as the multiplevalued function of average flux, or a single-valued function with $c \neq 0$ or $b \neq 1$, implies some kind of "cooperation" among the external drives with different values of $\left\{p_{i}\right\}$. As a specific example, the flux-fluctuation behavior observed in the microchip system shown in Fig. 1 with $a=-0.427, b=0.667$, and $c=0$ can be interpreted as due to the system's being under multiple external drives with different values of $\left\{p_{i}\right\}$.

In real physical systems, correlations among the external drives $M_{I}$ may improve the complexity of the flux fluctuation relationship. For example, both atmospheric precipitation and ice/snow melting can contribute to the flow of a river, but both drives may depend on the temperature. An urban traffic system can be driven by public traffic and other specific traffic demands, both being affected by the daily rhythmic behavior of human activities. From a more general perspective, the external drives with large positive correlation can be regarded as one effective single drive $M$ with $M_{J}$ being the weight to combine $\left\{p_{i}\right\}$, i.e., $p_{i}=\sum_{J} p_{i J} M_{J} / \sum_{J} M_{J}$, where $p_{i}$ characterizes the share of the whole drive $M=\sum{ }_{J} M_{J}$ on node $i$.

1. de Menezes, M. \& Barabási, A. L. Fluctuations in network dynamics. Phys. Rev. Lett. 92, 028701 (2004).

2. Eisler, Z. \& Kertész, J. Random walks on complex networks with inhomogeneous impact. Phys. Rev. E 71, 057104 (2005).

3. Duch, J. \& Arenas, A. Scaling of fluctuations in traffic on complex networks. Phys. Rev. Lett. 96, 218702 (2006).

4. Yoon, S., Yook, S.-H. \& Kim, Y. Scaling property of flux fluctuations from random walks. Phys. Rev. E 76, 056104 (2007).

5. Kujawski, B., Tadii, B. \& Rodgers, G. J. Preferential behaviour and scaling in diffusive dynamics on networks. New J. Phys. 9, 154-154 (2007).

6. Meloni, S., Gómez-Gardees, J., Latora, V. \& Moreno, Y. Scaling breakdown in flow fluctuations on complex networks. Phys. Rev. Lett. 100, 208701 (2008).

7. Zhou, Z. et al. Universality of flux-fluctuation law in complex dynamical systems. Phys. Rev. E 87, 012808 (2013).

8. Zhou, Z., Huang, Z.-G., Yang, L., Xue, D.-S. \& Wang, Y.-H. The effect of human rhythm on packet delivery. J. Stat. Mech. Theory E. 2010, P08001 (2010).
9. Bialek, W. et al. Statistical mechanics for natural flocks of birds. Proc. Natl. Acad. Sci. USA 10, 1073 (2012)

10. Ritort, F. Single-molecule experiments in biological physics: methods and applications. J. Phys.: Condens. Matter 18, R531 (2006).

11. Zhang, H., Tian, X.-J., Mukhopadhyay, A., Kim, K. S. \& Xing, J. Statistical mechanics model for the dynamics of collective epigenetic histone modification. Phys. Rev. Lett. 112, 068101 (2014).

12. Barabási, A. L. \& Albert, R. Emergence of scaling in random networks. Science 286, 509-512 (1999).

13. Echenique, P., Gómez-Gardees, J. \& Moreno, Y. Improved routing strategies for internet traffic delivery. Phys. Rev. E 70, 056105 (2004).

14. Echenique, P., Gómez-Gardees, J. \& Moreno, Y. Dynamics of jamming transitions in complex networks. Europhys. Lett. 71, 325-331 (2005).

15. Erdos, P. \& Renyi, A. On the evolution of random graphs. Bull. Inst. Internat. Statist 38, 343-347 (1960).

16. Watts, D. J. \& Strogatz, S. H. Collective dynamics of 'small-world' networks. Nature 393, 440-442 (1998).

17. Yan, G., Zhou, T., Hu, B., Fu, Z.-Q. \& Wang, B.-H. Efficient routing on complex networks. Phys. Rev. E 73, 046108 (2006).

18. Kishore, V., Santhanam, M. S. \& Amritkar, R. E. Extreme events on complex networks. Phys. Rev. Lett. 106, 188701 (2011).

19. Chen, Y.-Z., Huang, Z.-G. \& Lai, Y.-C. Controlling extreme events on complex networks. Sci. Rep. 4, 6121 (2014)

\section{Acknowledgments}

We thank Dr. DeMenezes for providing the microchip data. This work was partially supported by the NSF of China under Grant Nos. 11135001, 11275003. Y.C.L. was supported by ARO under Grant No. W911NF-14-1-0504.

\section{Author contributions}

Z.G.H., L.H. and Y.C.L. devised the research project. J.Q.D. performed numerical simulations. Z.G.H. and J.Q.D. did theoretical analysis. Z.G.H., Y.C.L. and L.H. wrote the paper.

\section{Additional information}

Competing financial interests: The authors declare no competing financial interests.

How to cite this article: Huang, Z.-G., Dong, J.-Q., Huang, L. \& Lai, Y.-C. Universal flux-fluctuation law in small systems. Sci. Rep. 4, 6787; DOI:10.1038/srep06787 (2014).

This work is licensed under a Creative Commons Attribution-NonCommercialNoDerivs 4.0 International License. The images or other third party material in this article are included in the article's Creative Commons license, unless indicated otherwise in the credit line; if the material is not included under the Creative Commons license, users will need to obtain permission from the license holder in order to reproduce the material. To view a copy of this license, visit http:// creativecommons.org/licenses/by-nc-nd/4.0/ 